\title{
Game Theoretic Frequency Reuse Approach in OFDMA Femtocell Networks
}

\author{
Joydev Ghosh and Dushantha Nalin K. Jayakody
}

\begin{abstract}
In this paper, we initially dealt with the issue of spectrum allocation among macro (or "licensed") and Femto (or "unlicensed") users in an orthogonal frequency division multiple access (OFDMA) femtocell network of non-cooperative game theoretic frequency reuse approach. We formulate the difficulty based on spectrum bidding. Here individual Secondary Users (SU) create an auction for the amount of bandwidth and every PU can share the frequency band among SUs by itself according to the intelligence from SUs without lowering its own performance. Here, we consider that the bidding is a non-cooperative game and one of its solutions is a Nash Equilibrium (NE). The femto base stations (FBSs) are grouped into different cluster for mitigating the undesired interference among them. The game theoretical method deals with the inter-cluster frequency clashes. We exemplified a link between utility function and the number of players by non-cooperative game theoretic approach to guide the spectrum sharing decision at the cell edges. The convergence of the development mechanism is rigorously scrutinized and extensive numerical outcomes are presented to illustrate their potential merits.
\end{abstract}

Index Terms-Femtocell Resource Allocation, Non-cooperative Game Theory, Nash-equilibrium (NE), Cumulative Distribution Function (CDF).

\section{INTRODUCTION}

A non-cooperative game has three basic elements: A set of players, a set of strategies, and a set of payoffs or utilities to a provided set of actions [1]. Players are the things with distinct and independent existence who make the decisions. Strategies are a set of authoritative orders to make a decision that indicates an action for a player. The payoff of a player exhibits the gratification level of a player for a prescribed strategy. The gratification level of the players is normally defined by a utility function. In game theory, a solution idea by means of the steady-state condition of non-cooperative game is named as a NE. The use of NE idea is essential in game theory to review the end result of the strategic interaction of many resolution creators. Additionally, we incorporate the spectrum substitutability metric which interprets the capability of the secondary service to switch among the operating frequency spectra provided by different primary services. Depending upon the cooperation between players, a game may be categorized in two groups as non-cooperative and cooperative games [2]. In a non-cooperative game, each player proceeds as a separate rational entity to make the best use of its payoff and take decisions independently. However, in cooperative games, all players in a crew act as a one entity. Players are not having

Joydev Ghosh and Dushantha Nalin K. Jayakody are with School of Computer Science and Robotics, National Research Tomsk Polytechnic University, RUSSIA, Email: joydev.ghosh.ece@gmail.com and nalin@tpu.ru. any competition between each other and do aim to maximize the total payoff of the crew. In [4], an approach for joint subchannel allotment, different adaptive modulation, and power control for multi-cell OFDMA based cognitive radio (CR) network is presented utilizing non-cooperative game. A virtual referee is included for the betterment of performance of the NE states. This referee has the authority to reshape the rule of the resource competition game for better resource allocation. In [5], a channel maximized and the power control module is introduced based on the NE approach for an OFDMA CR network. In [1], a non-cooperative game based on utility function is presented for femtocell signal to interference plus noise ratio (SINR) adjustment. The admission control which regulates the access policy is treated as an analytical issue in this scenario. For those UEs which are nearby adjacent FBS and not attach to it, they will create large interference to the femtocell for accessing the macrocell.

Here, we consider spectrum resource allocation in an OFDMA downlink (DL) network that composes of a macrocell and number of femtocells. By integrating cognitive potentials into these self-optimized FBSs, the cognitive-femtocell goal to increase the spectrum efficacy by using the unused spectrum when mitigating interference to the MBS in a spectrum overlay OFDMA based cognitive femtocell networks [30]. The unit of frequency resource to be assigned in an OFDMA CR network is called a resource block (RB) and it is consist of 14 subcarriers at a $25 \mathrm{kHz}$ spacing. Provided the RB utilization of the FBSs, the competitiveness for the spectrum in order to function effectively among FBSs can be developed in a gametheoretic configurations [3].

There are many importance on the deployment of femtocells in regard to the frequency reuse for mitigating the interferences between the different tiers so as to enhance the network performance [28]. An OFDMA (both LTE and WiMAX) is the great amount of dynamically assigned time and frequency slots [27], [31]. This significant grow in the modulability of resource allotment is both a advantage and a drawback. As orthogonal resources possible to assign to nearby pico and macrocells by femtocells, hence the feasibility of fine-tuned interference management prevails, which is non-feasible in case of GSM or CDMA. Although this needs a great amount of coordination. In this connection, an indispensable scheme is fractional frequency reuse (FFR) [25], whereby spectrum resources can be semi-statically alloted to indoors, cell edge area, or femtocell users, with power control on top to lower the throughput disparities experienced in each of these scenarios. Alternatively, a semi-static partition could simply be made 4 between femtocells and macrocells. The results in [29] indicate 
that even with dense femtocell deployments, most resources should go to the macrocell, since each femtocell only needs a small number of resource blocks to provide comparably high throughput to their user(s).

The proposed framework differs from the conventional cellular scheme in many aspects, for example the way to deploy a network, the technique to handle the interference and the condition to compute the network efficiency. Therefore, the cellular spectrum was regularly reused in space for continuous service provisioning over a large geographical area. The main goal of network coverage extension has led to few general characteristics over the 1-3G cellular standards such as circuitswitched channelization in a cell, regular cell planning and rigorous network configuration for interference management.

An interference management (IM) scheme femtocell deployment is essentially a spectrum reuse approach in an underlay macrocell network. Hence, how to reuse the spectrum in order to maximize the spectral efficiency is the basic issue to resolve. In $1-3 \mathrm{G}$, the optimum spectrum reuse is generally measured by a metric known as frequency reuse factor (FRF). The value of this factor determines the spatial separation of the co-channel cells based on the tolerable interference power on a mobile terminal.

Here, We study the proposed spectrum reuse scheme, where the idea is to implement an adaptive channel application on the location of the femtocells within a macrocell. A femtocell recognizes an interference signature from the network and intelligently reuses the proper channel modes to minimize the interference to the network. Environment perception and interference recognition are necessary procedures to facilitate the cognitive interference management and the resource allocation. It can be seen that the cognitive IM scheme provides the SUs the better SINR performance by allocating the most favorable spectrum resource to them without inclusion of PUs. As a consequence, it can be seen that it provides PUs the poor SINR performance. This can also be seen that the proposed scheme also effectively protects PUs nearby at an expense of marginal degradation in SU's SINR.This is mainly because femtocell can allocate better sub-channels to its serving SUs whilst neglecting the utilization of frequency allocation occupied by PUs nearby appling channel sensing.

The salient features of PUs and SUs in the CR is put to resolve the prioritized access matter for the dual-tier network. The CR mechanism proposed in [7] to maximize the throughput of Femto users under power control strategy is embraced in this paper. Here, we assume the resource allocation of femtocells by employing fractional frequency reuse (FFR) scheme [6], [24] to optimize the throughput while reducing interference to macro-cell users nearby [18]. With the help of spectrum sensing techniques, femtocells will get the potential of finding the available sub-bands. Specifically, the main contributions of this paper are:

1) We review the clustered pilot mechanism by a noncooperative game to stimulate the connection activities of non-subscribers within the network coverage of femtocell base station.

2) We consider that SUs are selfish and it also depicts that this bidding is a non-cooperative game in which individual SU reacts rationally to optimize utility. In this application of non-cooperative game, NE as an essential follows up and scrutinizes the salient characteristics of its end results. Our purpose of this spectrum sharing is to make the best use of the profit for SUs of using the fundamental of equilibrium.

3) The utility function explicated based on the channel capacity of the FBS network to achieve NE condition for an OFDMA based cognitive femtocell networks.

4) We present the adaptation of target rates per sub-channel (i.e., modulation coding schemes (MCS)), power control and access policies on the performance of the femtocell network. Access control policies are demonstrated for femtocell network by utilizing game theory technique where the macrocells and femtocells use different sets of sub-channels or share the total bandwidth under both open and closed access mechanisms. The quality of service (QoS) conscious access control design for the OFDMA femtocell network is conducted.

5) The CR based spectrum allocation is done by means of spectrum sensing to maximize the throughput of a femtocell. Here, we choose the detection process in the existence of the macro users by employing conventional hypothesis testing. We have chosen $H_{o}$ and $H_{1}$, respectively, to indicate hypothesis corresponding to the nonexistence and the existence of macro users.

This paper is organized as follows: The system model as well as analytical view of the paper are defined in Section II. In Section III, we present the simulation model to compute the network performance. Finally, results and discussions are provided in Section IV before concluding remarks in Section V.

\section{SYSTEM MODEL}

We contemplate a macrocell area with a number of femto base stations (FBSs) deployed at indoors within an OFDMA network. By integrating the cognitive capacities into FBSs, the spectrum possession nature of the macro base station (MBS) can be composed as a primary base station (PBS) and FBSs can be composed as secondary base stations (SBSs). Because of the selfish behaviour of the BS, the competitiveness for the usual spectrum resource among SBSs has been composed applying a game-theoretic foundation [26].

We establish a resource allocation model, as shown in Fig. 1, on the CR based femtocell cluster model where portable users of both the layers share the frequency band that incorporate $S$ sub-bands. Suppose there are $N_{f}$ FUEs taking service from $(n-1)$ FBSs, which are under the aegis of one macrocell providing network service to $N_{m}$ MUEs. Let us we consider $U_{n}$ be the set of portable user equipments in the $n^{t h}$ femtocell, which means users are getting network facility from $n^{\text {th }}$ BS of the respective layer. For expediency, suppose $\mathcal{U}_{m} \triangleq \mathcal{U}_{1}$ comprises the set of MUEs and $\mathcal{U}_{f} \triangleq \bigcup_{i=2}^{n} \mathcal{U}_{n}=\left\{N_{m}+1, \cdots, N_{m}+N_{f}\right\}$ indicate the set of total FUEs. Moreover, $\mathcal{U}$ and $\mathcal{B}$ be the sets of total portable user equipments and service network entity, respectively. Therefore, we get $\mathcal{U}=\mathcal{U}_{m} \cup \mathcal{U}_{f}=\{1,2, \cdots, N\}$ 
and $B=\{1,2,3, \cdots, n\}$ where $1^{\text {st }} \mathrm{BS}$ is selected to be the MBS and rest $B_{f}=\{1,2,3, \cdots,(n-1)\}$ indicates the set of all FBSs. For the sake of analysis, we consider $b_{l} \in \mathcal{B}$ to symbolize the BS which serves portable UE $I$ and let $\mathcal{S}=\{1,2,3, \cdots, S\}$ be the set of total orthogonal subchannels.
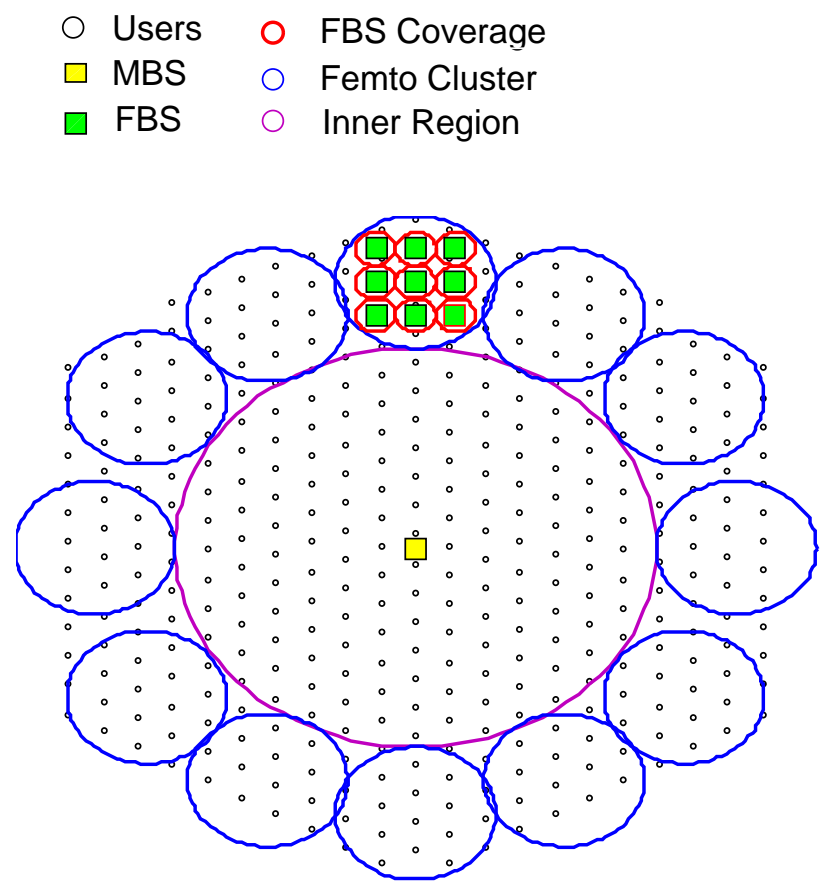

Fig. 1: System model of two- tier macrocell-femtocell network

Let $A_{t}^{k}=\left\{a_{t}^{k}(1), \ldots . a_{t}^{k}\left(N_{S B S}^{t}\right)\right\} \in \mathcal{P}_{k}$ indicate the action of the $k$-th SBS over entire accessible resource blocks (RBs) to SBS at time $t, \mathcal{P}_{k}$ with indicating the action space of the $k$-th SBS. $\left.A^{t}=\left\{a_{t}^{1}, \ldots . a_{t}^{k}\right)\right\} \in \mathcal{P}$ is considered to indicate the formation of the actions from the entire SBSs at time $t$. $\mathcal{P}$ is the joint action space of the entire SBSs. The resource allotment technique in the cognitive femtocell network follows a time slotted network model where every time slot length equals to that of a RB $(0.6 \mathrm{~ms})$, with RB being the smallscale unit of resource that to be assigned. Let $t \in 1,2, \ldots, T$ indicate the time slot index where $\mathrm{T}$ stands for the time horizon of the developed game.

Let $q_{k}^{f t} \in\left\{1,2, \ldots, Q_{s}\right\}$ used to indicate channel quality state of the $k$-th SBS on the $f$-th RB at time $t$ after quantization. For instance, the channel quality can be achieved by quantizing an instantaneous valued channel model consisting of circularly symmetric complex Gaussian random variables into different states.

To provide a detailed account in the subchannel allotments (SA), suppose $\mathbf{C}=\mathbf{R}^{N \times \mathcal{U}}$ be the SA matrix for all MUEs over $S$ sub-bands where

$$
\begin{aligned}
\mathbf{C}(i, s)= & \sum_{f=1}^{N_{S B S S}^{t}} B W \times \log _{2}\left[1+\frac{a_{k}^{f t} \times q_{k}^{f t}}{I_{i k}^{f s}}\right] \\
& =x_{i}^{s}= \begin{cases}1, & \text { If subchannel } s \text { allocates } \\
& \text { for UE } i ; \\
0, & \text { Otherwise. }\end{cases}
\end{aligned}
$$

We consider that the assignment of a sub-band should not be more than one portable UE in any cell. Then, we have

$$
\sum_{i \in \mathcal{U}_{n}} x_{i}^{s} \leq 1, \quad \forall n \in \mathcal{B} \text { and } \forall s \in \mathcal{S} .
$$

These conditions apply to all resource allocation issues studied in this paper. The utility function of $i^{\text {th }}$ femto user (FU) can be defined as [20]:

$$
I_{i k}^{f s}=\sigma_{i}^{2}+\sum_{i=1, k=\mathcal{U}_{m}}^{n} w_{i k}^{f 2 m, s} \times \mathbf{S}_{-i},
$$

The elements of this interference matrix, i.e., $w_{i k}^{f 2 m, s}$ where $i, k \in \mathcal{B}$, indicate the cross channel quality between $i^{t h}$ users BS and $j^{t h}$ users BS. Considering channel reciprocity $w_{i}^{f 2 m}$ is a symmetric matrix, i.e $w_{i k}^{f 2 m, s}=w_{k i}^{f 2 m, s}$. Thus, it is intelligible that the interference matrix has zero-valued diagonal elements, $w_{i i}^{f 2 m, s}=0$ for $\forall i \in \mathcal{B}$. We make use of $S_{i} \in 0,1$ to indicate the action of the $k^{t h}$ BS $(\mathcal{B}=\{1,2,3, \cdots, k, \cdots, n\})$ over the $s^{t h}$ sub-band, where 0 indicates not to transmit and 1 indicates to transmit. Let us assume the action profile as $\left(s_{i}, s_{-i}\right)$ where $s_{i}$ and $s_{-i}$ for the element of $\mathbf{S}_{i}$ and $\mathbf{S}_{-i}$, respectively. The set $\mathbf{S}_{-i}=\Pi_{j \neq i} \mathbf{S}_{j}$ is symbolized as the action profiles except $i$.

The objective of SU is to optimize the utility function by a proper power allotment [19]. Therefore, we can formulate an optimization issue as [14]:

$$
\max _{P_{i}^{s}} U_{i}^{f}\left(P_{i}^{f}, P_{-i}^{f}\right),
$$

s.t:

$$
\begin{aligned}
& 0 \leq P_{i}^{f s} \leq P_{\text {max }}, \\
& 0 \leq \sum_{j \cong-i \in \mathcal{U}_{f}} P_{-i}^{f s} \leq P_{\text {max }},
\end{aligned}
$$

where $\mathbf{P}_{i}^{f}=\left\{P_{i}^{f 1}, P_{i}^{f 2}, \cdots, P_{i}^{f n}\right\}$ is $i^{\text {th }}$ femto users power allocation vector, while $\mathbf{P}_{-i}^{f}$ gives a detail account in words of all the other SUs belonging to the same femto base station network coverage, $P_{i}^{f s}$ is the $i^{\text {th }}$ femto user allocated power to the $s^{t h}$ sub-channel, $G_{s i}^{f s}$ is the channel gain between the $i^{t h}$ femto users BS and i-th FUs receiver over the s-th subchannel.

The sub-channel, $G_{j i}^{f s}$ and $G_{i k}^{f m}$ are the channel gain between $i^{\text {th }}$ FUs BS and $j^{\text {th }}$ femto user over $s^{\text {th }}$ sub-channel and between $i^{\text {th }}$ femto users BS and $k^{\text {th }}$ macro user, respectively, $\lambda^{f s}$ is the price per unit of power for sharing the $s^{\text {th }}$ sub-channel. Note that $\beta_{i}^{f s}=1$ if subchannel $s$ is assigned to the $i^{t h} \mathrm{FU}$ in $n^{t h}$ femtocell and $\beta_{i}^{f s}=0$ otherwise. $I_{i k}^{f 2 m, s}$ represents $i^{t h}$ FUs received interference 


$$
\begin{aligned}
U_{i}^{f} & =\sum_{s \in \mathcal{B}, k \in \mathcal{U}_{m}, i \in \mathcal{U}_{f}} \mu_{i}^{f s} \\
& =\sum_{s \in \mathcal{B}, k \in \mathcal{U}_{m}, i \in \mathcal{U}_{f}} \beta_{i}^{f s}\left[\log _{2}\left(1+\left(\frac{P_{i}^{f s} G_{i i}^{f s}}{\sum_{j \neq i, j \in \mathcal{U}_{f}} P_{j}^{f s} G_{j i}^{f s}+I_{i k}^{f s}+\Delta f N_{0}}\right)\right)-\lambda^{f s}\left(P_{k}^{m s} G_{i k}^{f 2 m, s}\right)\right],
\end{aligned}
$$

from the $k^{t h} \mathrm{MU}$.

Hence, SINR of $k^{t h}$ MUE can be expressed as:

$$
\gamma_{k}^{m s}=\left\{\begin{array}{l}
\frac{P_{k}^{m s} G_{k 2 m}^{m s}}{\sum_{i \in \mathcal{U}_{f}} P_{i}^{f s} G_{v 2 m}^{f s}+\sigma_{i}^{2}+\Delta f N_{0}}, \text { FUE } i \text { exists } \\
\frac{P_{k}^{m s} G_{k 2 m}^{m s}}{\sigma_{i}^{2}+\Delta f N_{0}}, \quad \text { FUE i does not exist. }
\end{array}\right.
$$

The parameter $P_{i}^{f s}$ represents transmission power of $j^{\text {th }} \mathrm{SU}$ in the $s^{t h}$ sub-channel.

Hence, SINR on $s^{t h}$ sub-band of $i^{t h}$ femto user can be written as:

$$
\gamma_{i}^{f s}= \begin{cases}\frac{P_{i}^{f s} G_{s i}^{f s}}{\sum_{j \neq i, j \in \mathcal{U}_{f}} P_{j}^{f s} G_{j i}^{f s}+I_{i k}^{f s}+\Delta f N_{0}}, & \text { MUE k exists } \\ \frac{P_{i}^{f s} G_{s i}^{f s}}{\sum_{j \neq i, j \in \mathcal{U}_{f}} P_{j}^{f s} G_{j i}^{f s}+\sigma_{i}^{2}+\Delta f N_{0}}, & \text { MUE k does not exist. }\end{cases}
$$

Let, $\gamma_{o}^{f}$ be the target SINR of the poorest quality of service getter FUE $i$ whose distance from its associated FBS is certainly $d_{f}$. Hence, the outage probability constraint can therefore be written as [22]:

$$
P_{r}\left\{\gamma_{i}^{f s}\left(d_{f}\right)<\gamma_{0}^{f s}\right\}<p_{o}^{f s},
$$

where $p_{o}^{f s}$ indicates the target outage probability. The corresponding distribution function (CDF) is expressed by:

$$
\Psi\left(\gamma_{i}^{f s}\right)=1-\frac{1}{2} \operatorname{erfc}\left(\frac{10 \log _{10} \gamma_{0}^{s}-v_{i}}{\sqrt{2} \eta_{i}}\right),
$$

where $\operatorname{erfc(.)}$ is considered to denote complementary error function, defined as $\operatorname{erfc}(x)=1-\operatorname{erf}(x)=e^{-x^{2}} \operatorname{erfcx}(x)$ in which $\operatorname{erfcx}(x)$ is used to avoid arithmetic underflow for better estimation.

\section{Resource Allocation in femtocell cluster by Spectrum Sensing:}

The occupancy of $n^{\text {th }}$ sub-band is feasible to detect with the help of a hypothesis test can be expressed as [12]:

$$
V^{\prime}=\left\{\begin{array}{lc}
N_{n} ; & \mathcal{H}_{o, n} \\
S_{n}+N_{n} ; & \mathcal{H}_{1, n},
\end{array}\right.
$$

where $n=\left\{1,2, \cdots, N_{s c}\right\}, S_{n}$ and $N_{n}$ indicate the discrete frequency response of $s(t)$ and $n(t)$, respectively. The end result of the integrator is supposed to be the test statistic $V^{\prime}$ over a period of $T$. We shall find its suitability to quantify the false alarm and detection probabilities by utilizing the related aggregate as [23]:

$$
V^{\prime}=\left(\frac{1}{N_{0}}\right) \int_{0}^{T} y^{2}(t) d t
$$

Any interval of duration $T$ will serve as a sampling interval based on its selection.

Under the hypothesis $\mathcal{H}_{o}$, the test statistic $V^{\prime}$ can be written as:

$$
V^{\prime}=\sum_{i=1}^{2 T W} N_{n}^{2},
$$

where $N_{n}=\frac{A_{n}}{\sqrt{2 W N_{02}}}=$ normalized noise sample value and $A_{n}=$ noise sample value. Here, $V^{\prime}$ is a chi-square distribution with $2 T W$ degrees of privilege, for which extensive table exists in [10]. Under the hypothesis $\mathcal{H}_{1}$, the test statistic $V^{\prime}$ can be written as:

$$
V^{\prime}=\sum_{i=1}^{2 T W}\left(S_{n}+N_{n}\right)^{2}
$$

The sum in (14) having a non-central chi-square distribution with $2 T W$ degrees of privilege and a non-centrality variable $\lambda$ can be given by $\lambda=\frac{E_{s}}{N_{02}}$.

The probability of false alarm $Q_{o}$ for given threshold $V_{T}^{\prime}$ can be written by

$$
\begin{aligned}
Q_{0} & =\operatorname{Prob}\left\{V^{\prime}>V_{T}^{\prime} \mid \mathcal{H}_{o}\right\}=\operatorname{prob}\left\{X_{2 T W}^{2}>V_{T}^{\prime}\right\} \\
& =\frac{1}{2} \operatorname{erfc}\left[\frac{V_{T}^{\prime}-2 T W}{2 \sqrt{2} \sqrt{T W}}\right] .
\end{aligned}
$$

For the same threshold level $V_{T}^{\prime}$, the probability of detection $Q_{d}$ can be expressed by:

$$
\begin{aligned}
Q_{d} & =\operatorname{Prob}\left\{V^{\prime}>V_{T}^{\prime} \mid \mathcal{H}_{1}\right\}=\operatorname{prob}\left\{x_{2 T W}^{2}(\lambda)>V_{T}^{\prime}\right\} \\
& =\frac{1}{2} \operatorname{erfc}\left[\frac{V_{T}^{\prime}-2 T W-\lambda}{2 \sqrt{2} \sqrt{T W+\lambda}}\right] .
\end{aligned}
$$

The symbol $x_{2 T W}^{2}(\lambda)$ represents a non-central chi-square parameter with $2 T W$ degrees of privilege. The channel throughput of a SU $i$ can be obtained for the spectrum assignment of $b_{i}$ as follows:

$$
C_{i}=b_{i} \log _{2}\left(1+\alpha_{i} \gamma_{i}\right),
$$

where $\alpha_{i}=\frac{1.5}{\left(\frac{\ln 0.2}{B E R_{i}^{t a r}}\right)}$. To provide guaranteed transmission maintaining quality, BER should be within the target level (i.e. 
$B E R_{i}^{t a r}$ ) [8]. For the PU, we introduce the costing function to charge the SUs can be expressed by:

$$
e(S)=x+y\left(\sum_{j} s_{j}\right)^{\tau}
$$

where $x, y$ and $\tau$ are non-negative constants, 1 and the strategy of individual players to the allotted spectrum size (symbolized by $s_{j}$ for $\mathrm{SU} j$ ) is non-negative.

Let, $w$ indicates the price of the spectrum for the PU. Hence, the necessary criterion $C(S)>w \times \sum_{j} s_{j}$ is to confirm that the PU is prepared to allotted spectrum of size $s$ with the SUs. The revenue of the SU $i$ to be achieved from $r_{i} \times C_{i} \times s_{i}$ whilst the price of spectrum assignment is $s_{i} C(\mathbf{S})$. Hence, the profit of the SU $i$ can be achieved as follows:

$$
\rho_{i}(\mathbf{S})=r_{i} C_{i} s_{i}-s_{i} e(\mathbf{S}) .
$$

Therefore, the profit can be re-expressed as follows:

$$
\rho_{i}(\mathbf{S})=r_{i} C_{i} s_{i}-s_{i}\left(x+y\left(\sum_{j} s_{j}\right)^{\tau}\right) .
$$

The minimal profit function for $i^{t h} \mathrm{SU}$ can be obtained from

$$
\frac{\partial \rho_{i}(\mathbf{S})}{\partial s_{i}}=r_{i} C_{i}-\left[\left(x+y\left(\sum_{j} s_{j}\right)^{\tau}\right)+s_{i} y \tau\left(\sum_{j} s_{j}\right)^{\tau-1}\right]
$$

Let $\mathbf{S}_{-i}$ denote the set of strategies embraced by all except $i^{\text {th }} \mathrm{SU}$ (i.e., $\mathbf{S}_{-i}=\left(\left.s_{j}\right|_{(j=1,2, \cdots, N) ; j \neq i}\right)$ and $\mathbf{S}=\mathbf{S}_{-i} \cup \mathbf{S}_{i}$. The optimum response function of SU $i$ provided the assigned spectrum size of other SUs $s_{j}$, where $j \neq i$ is written below [13], [15]:

$$
\mathcal{A}_{i}\left(\mathbf{S}_{-i}\right)=\arg \max _{s_{i}} \rho_{i}(\mathbf{S}) \text {. }
$$

The set $\mathbf{S}^{*}=\left\{s_{1}^{*}, s_{2}^{*}, \cdots, s_{N}^{*}\right\}$ represents the $\mathrm{NE}$ of the game.

$$
s_{i}^{*}=\mathcal{A}_{i}\left(\mathbf{S}_{-i}^{*}\right), i
$$

here $S_{-i}^{*}$ represents the set of optimum responses of SU $i$ for $j \neq i$. Therefore, to achieve NE [1], we have to solve the following set of equations:

$$
\frac{\partial \rho_{i}(\mathbf{S})}{\partial s_{i}}=r_{i} C_{i}-\left[x+y\left(\sum_{j} s_{j}\right)^{\tau}+s_{i} y \tau\left(\sum_{j} s_{j}\right)^{\tau-1}\right]
$$

We construct an optimization issue with the intension defined as follows:

$$
\text { Minimize }: \sum_{i=1}^{N}\left|s_{i}-\mathcal{A}_{i}\left(\mathbf{S}_{-i}^{*}\right)\right|
$$

The correlation between the allocated spectrum size in the present and next iteration can be given by [17]:

$$
s_{i}(t+1)=s_{i}(t)+a_{i} s_{i}(t) \frac{\partial \rho_{i}(\mathbf{S})}{\partial s_{i}(t)},
$$

where $s_{i}(t+1)$ is the assigned spectrum size at instant $t$ and $a_{i}$ is the learning rate of a $i^{t h}$. By considering the condition, $\tau=1, s_{i}(t+1)$ can be again re-expressed as in (27). Hence, the repeated Cournot game is to be represented in the form of matrix as follows [9]:

$$
s(t+1)=\mathcal{N}\{s(t)\} .
$$

At the equilibrium, we are having $s(t+1)=s(t)=s$, viz. $s=N(s)$ where $N$ is the self-mapping function of fixed point $s$. The fixed point for pricing function is to be achieved by resolving the number of equations:

$$
a_{i} s_{i}(t)\left(r_{i} C_{i}-x-2 s_{i} y-y \sum_{j \neq i} s_{j}\right)=0, \forall i
$$

With two SUs in the CR scenario, we have fixed points $s_{0}, s_{1}, s_{2}, s_{3}$ which can be written as follows [3]:

$$
\left\{\begin{array}{l}
s_{0}=(0,0) ; s_{1}=\left(\frac{r_{1} C_{1} s_{1}-x}{2 y^{2}}, 0\right) ; s_{2}=\left(0, \frac{r_{2} C_{2} s_{2}-x}{2 y^{2}}\right) \\
s_{3}=\left(\frac{\left(r_{2} C_{2} s_{2}-2\left(r_{1} C_{1} s_{1}\right)+x\right.}{1-4 y^{2}}, \frac{r_{1} C_{1} s_{1}-2\left(r_{2} C_{2} s_{2}\right)+x}{1-4 y^{2}}\right)
\end{array}\right.
$$

where $s_{3}$ is the NE.

Note that the rooted point is stable if and only if the eigenvalues are located to inner part of the unit circle of the complex plane (i.e., $\lambda_{i}<1$ for $C=1,2,3, \cdots, N$ ) [9]. In this case, the Jacobian matrix can be defined as follows in [16]. Since this Jacobian matrix [16] is neither diagonal nor triangular, the characteristic equation to achieve the eigenvalues can be followed as in [16] and from there we can get the roots of general quadratic equations. Then, we probe the stability criteria for the fixed points $s_{0}, s_{1}, s_{2}$, respectively. The fixed point $s_{0}$ is called a boundary equilibrium [11] and have economic meaning when $r_{1} C_{1} s_{1}>x$ and $r_{2} C_{2} s_{2}>x$.

$$
s_{i}(t+1)=s_{i}(t)+a_{i} s_{i}(t) r_{i} C_{i}-\left[x+y\left(\sum_{j} s_{j}\right)^{\tau}+s_{i} y \tau\left(\sum_{j} s_{j}\right)^{\tau-1}\right]
$$




$$
\begin{aligned}
& \mathbf{J}\left(s_{1}, s_{2}\right)=\left[\begin{array}{cc}
1+a_{1}\left(r_{1} C_{1} s_{1}-x-4 y s_{1}-y s_{2}\right) & -y a_{1} s_{1} \\
-y a_{2} s_{2} & 1+a_{2}\left(r_{2} C_{2} s_{2}-x-4 y s_{1}-y s_{2}\right)
\end{array}\right] \\
& \mathbf{J}(0,0)=\left[\begin{array}{cc}
1+a_{1}\left(r_{1} C_{1} s_{1}-x\right) & 0 \\
0 & 1+a_{1}\left(r_{2} C_{2} s_{2}-x\right)
\end{array}\right] \\
& \mathbf{J}\left(0, \frac{r_{2} C_{2} s_{2}-x}{2 y^{2}}\right)=\left[\begin{array}{cc}
1+a_{1}\left(-r_{1} C_{1} s_{1}+x\right) & 0 \\
a_{2} \frac{r_{2} C_{2} s_{2}-x}{2} & 1+\frac{a_{2}}{2}\left(2 r_{2} C_{2} s_{2}-r_{1} C_{1} s_{1}-x\right)
\end{array}\right] \\
& \mathbf{J}\left(\frac{r_{1} C_{1} s_{1}-x}{2 y^{2}}, 0\right)=\left[\begin{array}{cc}
1+a_{1}\left(-r_{1} C_{1} s_{1}+x\right) & a_{1} \frac{r_{1} C_{1} s_{1}-x}{2} \\
0 & 1+\frac{a_{2}}{2}\left(2 r_{2} C_{2} s_{2}-r_{1} C_{1} s_{1}-x\right)
\end{array}\right] \\
& J\left(\frac{r_{2} C_{2} s_{2}-2\left(r_{1} C_{1} s_{1}\right)+x}{1-4 y^{2}}, \frac{r_{1} C_{1} s_{1}-2\left(r_{2} C_{2} s_{2}\right)+x}{1-4 y^{2}}\right)= \\
& {\left[\begin{array}{cc}
1+a_{1} \frac{r_{1} C_{1} s_{1}-2 y r_{2} C_{2} s_{2}+3 y r_{1} C_{1}-y x-x}{1-4 y^{2}} & -a_{1} \frac{r_{2} C_{2} s_{2}-2\left(r_{1} C_{1} s_{1}\right)+x}{1-4 y^{2}} \\
-a_{2} \frac{r_{1} C_{1} s_{1}-2\left(r_{2} C_{2} s_{2}\right)+x}{1-4 y^{2}} & 1+a_{2} \frac{r_{2} C_{2} s_{2}-2 y r_{1} C_{1} s_{1}+3 y r_{2} C_{2} s_{2}-y x-x}{1-4 y^{2}}
\end{array}\right],}
\end{aligned}
$$

The coordinate $(0,0)$ will be stable if $1+a_{1}\left(r_{1} C_{1} s_{1}-x\right)<1$ and $1+a_{2}\left(r_{2} C_{2} s_{2}-x\right)<1$. We come to the stability criterion for $s_{0}$ as follows:

$$
r_{1} C_{1} s_{1}<x \quad \text { and } \quad r_{2} C_{2} s_{2}<x
$$

These criteria implicit that whilst none of the SUs is ready to share the spectrum corresponds to fixed point $(0,0)$, the system is stable. It means whilst the price of the spectrum as proposed by the PU is larger than the revenue achieved from assigned spectrum, a SU is ready to stay out of the system. Hence, the conditions obtained in (36) for the stability of the fixed point is not obeying economic meaning. So that, fixed point $s_{0}$ never stable. For the fixed point $s_{2}$, we have the Jacobian matrix written as in (33). For the first eigenvalue, it is practicable to fulfil the criterion $\left\{1+a_{1}\left(-r_{1} C_{1} s_{1}+x\right)\right\}<$ $1\left\{r_{1} C_{1} s_{1}\right\}>x$. Although, we computed the condition for the second eigenvalue.

$$
1+\frac{a_{2}}{2}\left(2 r_{2} C_{2} s_{2}-r_{1} C_{1} s_{1}-x\right)<1 .
$$

As $x$ is a non-negative, we achieve $\left(2 r_{2} C_{2} s_{2}-r_{1} C_{1} s_{1}-\right.$ $x)<0$ or $2 r_{2} C_{2} s_{2}<r_{1} C_{1} s_{1}+x$. Although, this is impossible as NE can only be achieved from (30) if $r_{1} C_{1} s_{1}+r_{2} C_{2} s_{2}>x$. So that, fixed point $\left(0, \frac{r_{2} C_{2} s_{2}-x}{2 y^{2}}\right)$ is never stable. Similarly, fixed point $\left(\frac{r_{1} C_{1} s_{1}-x}{2 y^{2}}, 0\right)$ is never stable.

For Nash equilibrium point $s_{3}$, let us assume a CR scenario with one PU and two SUs using a spectrum of $25 \mathrm{MHz}$. The target BER for both types of users (PUs/SUs) is $B E R_{i}^{t a r}=$ $10^{-4}$. For the pricing function of PU, we consider $x=0$ and $y=1$, whilst $\tau$ is tuned depending upon the computation scenario (e.g. $\tau=1$ ) and the price of spectrum for PU is $w=1$. The revenue of a SU per unit transmission rate is $r_{i}=10, \forall i$ and $a_{1}=a_{2}=0.14$. Now the Jacobian matrix can be written as:

$$
\left\{\begin{array}{l}
j_{1,1}=1-\frac{0.28}{3}\left(2 r_{1} C_{1} s_{1}-r_{2} C_{2} s_{2}\right) \\
j_{2,2}=1-\frac{0.28}{3}\left(2 r_{2} C_{2} s_{2}-r_{1} C_{1} s_{1}\right) \\
\frac{j_{1}, 1+j_{2}, 2}{2}=1-\frac{0.28}{6}\left(r_{1} C_{1} s_{1}+r_{2} C_{2} s_{2}\right)
\end{array}\right.
$$

Table 1: MODULATION CODING SET (MCS) [12]

\begin{tabular}{|l|l|l|l|l|}
\hline Index & SINR range dB & $\begin{array}{l}\text { Modula- } \\
\text { tion }\end{array}$ & $\begin{array}{l}\text { Code } \\
\text { rate }\end{array}$ & $\begin{array}{l}\text { Bit } \\
\text { rate }\end{array}$ \\
\hline 1 & $-3.14 \leq S I N R<-0.73$ & QPSK & $\frac{1}{12}$ & 1.6 \\
\hline 2 & $-0.73 \leq S I N R<2.09$ & QPSK & $\frac{1}{6}$ & 3.2 \\
\hline 3 & $2.09 \leq S I N R<4.75$ & QPSK & $\frac{1}{3}$ & 6.4 \\
\hline 4 & $4.75 \leq S I N R<7.86$ & QPSK & $\frac{1}{2}$ & 9.6 \\
\hline 5 & $7.86 \leq S I N R<9.94$ & QPSK & $\frac{2}{3}$ & 12.8 \\
\hline 6 & $9.94 \leq S I N R<13.45$ & 16QAM & $\frac{1}{2}$ & 19.2 \\
\hline 7 & $13.45 \leq S I N R<18.6$ & 16QAM & $\frac{2}{3}$ & 25.6 \\
\hline 8 & $18.6 \leq S I N R<24.58$ & 64QAM & $\frac{2}{3}$ & 38.4 \\
\hline 9 & $24.58 \leq S I N R$ & 64QAM & $\frac{5}{6}$ & 48 \\
\hline
\end{tabular}

\section{Simulation MOdel}

To evaluate the performance of the proposed scheme, a simulation framework has been developed with the use of MCS sets in Table I on MATLAB. In particular, each simulation run is carried out according to the following steps.

1) The quantity of useful data bits is liable to vary, which is determined by the modulation coding scheme (MCS). The utilization of MCS is explicated here for the optimization of coverage probability. The choice of suitable MCS is dependent on the value of the receiver SINR. When the SINR equals to $7.0 \mathrm{~dB}$, the FBS uses QPSK $\frac{1}{2}$. The dissimilar MCS that are determined by the standard are the following: QPSK, 16QAM, 64QAM. The SINR value greater than $24.58 \mathrm{~dB}$ should satisfy a spectral efficiency of $48 \mathrm{bps} / \mathrm{Hz}$ with a MCS summarized in Table I adapted 
from IEEE 802.16e [21]. Normally, subscriber switches to a large energy efficient MCS if the SINR is high. The subscriber can also switch to even more robust MCS if the SINR is small. Once the MCS is determined, the amount of bits per symbol can be computed.

2) At every time period each player should form an expectation of the competitors outcomes in the next time interval in order to obtain the corresponding profit optimizing quantities for interval $t+1$. If we indicate by $s_{i}(t)$ the outcome of firm $i$ at time interval $t$, then its production $s_{i}(t+1)$, for $i=1,2$ for the interval $t+1$ can be achieved by solving the maximization issues. Firms attempt to utilize more complex expectations; hence they attempt to utilize local data based on the marginal profit $\frac{\partial s_{i}(t)}{\partial \rho_{i}(S)}$. At every time interval $t$ each firm either enhances or reduces its production $s_{i}(t)$ at the interval $(t+1)$ for the positive or negative marginal profit, respectively.

3) Here we proposed a search algorithm for Seeking the best strategy to deal with the SU's best response which is linearly increasing in price. In the text followed, we investigated how every SU obtains the NE in a distributed manner. For the sake of simplicity of calculation, the dependence on $s$ is neglected.

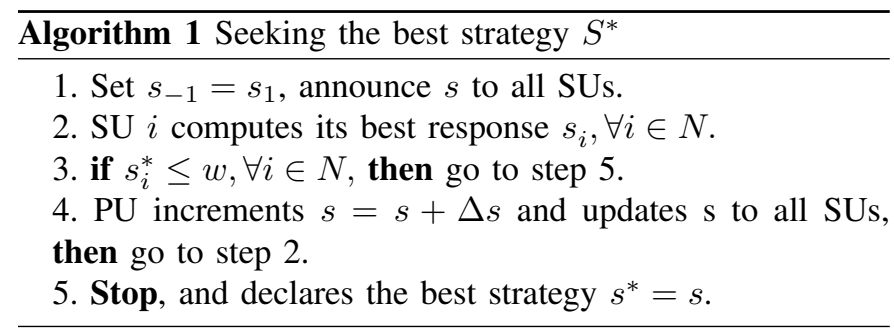

\section{RESULTS AND DISCUSSION}

Here, we illustrate interpretative numerical outcomes to justify the effectiveness of the introduced network model. In reference no. [16], Dusit Niyato et al. noticed that a less number of links at the primary service causes a destabilization effect. This is due to the less cost at the equilibrium. Higher values of learning rates produce large instability in price adaptation that diverges from the equilibrium when the price is less. This destabilization impact is also noticed whilst $y$ becomes high (i.e., for a less equilibrium price). Particularly, the stability regions in $\left(a_{1}, a_{2}\right)$ plane for different channel qualities are depicted in Fig. 2. If $a_{1}$ and $a_{2}$ are set of values in this region, the spectrum assignment can then undergo the stability and the NE shall be achieved, else the sharing shall be unstable, and fluctuations can occur. As anticipated, depending upon quality of channel, spectrum shared to the SUs. The stability region can be interpreted as a plane for that if the learning rates are considered into the plane, the distributed costing model shall converge to the solution and it is noticed that the speed of convergence depends largely on the learning rates. Here, we observe that the stability region of the distributed competitive pricing model for $\gamma_{1}=\gamma_{2}=7$ $\mathrm{dB}$ is much larger than that for each of the models where $\gamma_{1}=\gamma_{2}=8 \mathrm{~dB}$ and $\gamma_{1}=\gamma_{2}=10 \mathrm{~dB}$. Similarly, the pricing model for $\gamma_{1}=\gamma_{2}=8 \mathrm{~dB}$ is larger than that of model where $\gamma_{1}=\gamma_{2}=10 \mathrm{~dB}$. Although, a proper setting of the learning rate variable is needed to make sure that the spectrum allotment is stable and it is able to find the NE. Consequently, if the speed adjustment parameter $\left(a_{1}, a_{2}\right)$ is large enough, it causes fluctuations in the price adaptation and distributed competitive pricing scheme (process) may require large number of iteration to reach the equilibrium.

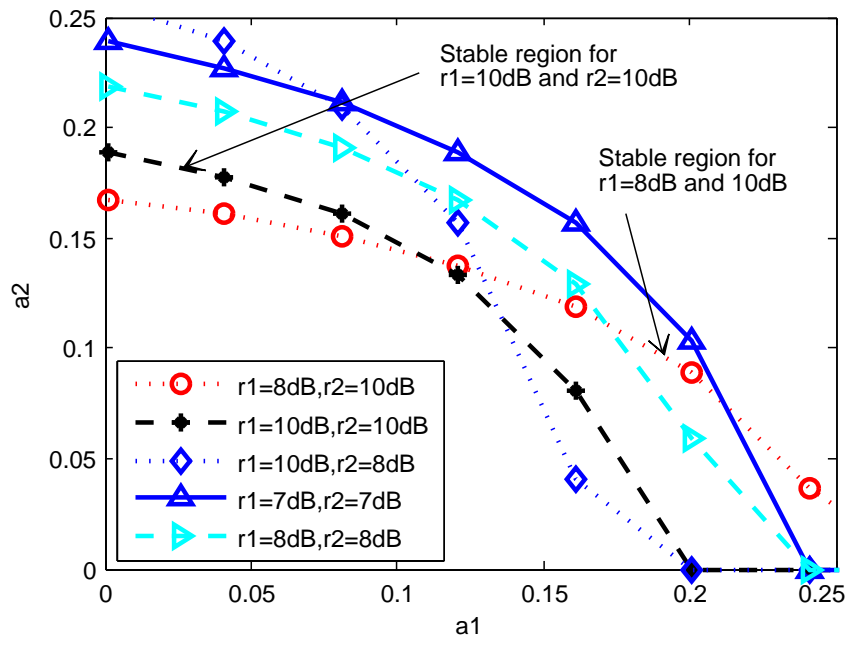

Fig. 2: NE stability region under different learning rates

In reference no. [16], the fig. 7 depicted distinctions in price under the different link conditions for the spectrum provided by the primary services. In this pricing model, when $\gamma_{1}$ grows, the respective spectrum stipulation becomes higher, and hence,the primary service can grow its offered price to achieve larger profit. In figs. 3, the parameter $y$ is also having a notable effect on the NE state. To investigate the effect of $y$, we set the learning rates corresponding to the spectrum offered by the secondary services as follows: $\mathrm{a} 1=\mathrm{a} 2=0.25$ and $\mathrm{a} 1=\mathrm{a} 2=0.1$. Precisely, whilst the secondary service capable of moving freely among the sub-bands provided by the primary services (i.e., $y$ is large), the NE results in a little charge. Hence the secondary service has the tendency to move to the low-priced spectrum simply, the order of competition among the primary services becomes higher. The learning rate $\left(a_{1}, a_{2}\right)$ also impacts the rates of increase and decrease in charges due to the spectrum substitutability factor. As SU capable to obtain the larger transmission rate from the same spectrum size because of the adaptive modulation, the larger profit can be obtained and as a result, the SUs would rather to have a larger spectrum size. when $\mathrm{y}=0$, a SU cannot change its operating spectrum. When $y=1$, the spectrum is superbly exchangeable and SU may move among the frequency spectra freely. As $y$ get larger, for the distributed resource allocation scheme, spectrum cost increases in Fig. 3(a). Interestingly, when the spectrum is not substitutable $(0 \leq y<1)$, the spectrum cost set to each of the SU by primary service to gain the highest profit. Moreover, when the spectrum is at exchangeable state, the SU may move to the other primary service providing the lowest price. Consequently, a situation 


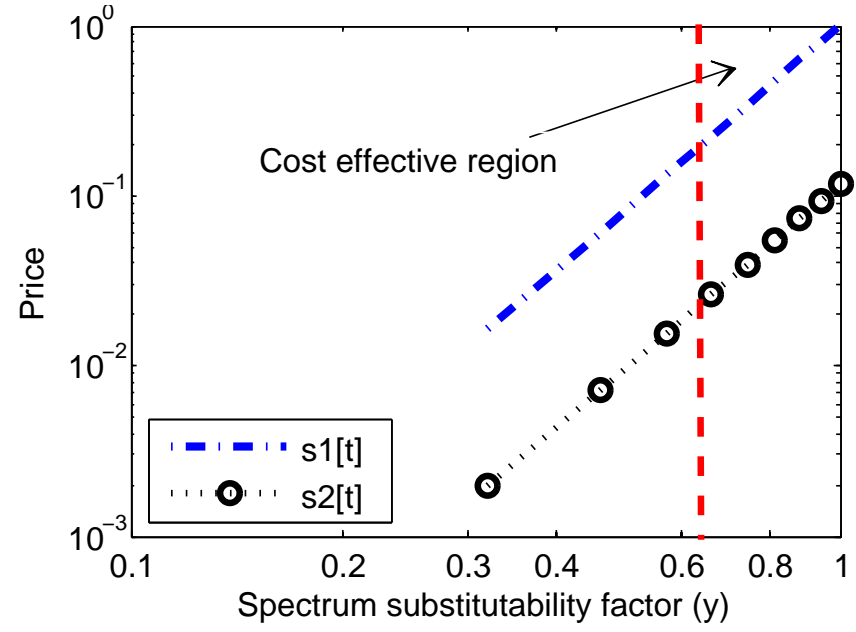

Fig. 3(a)

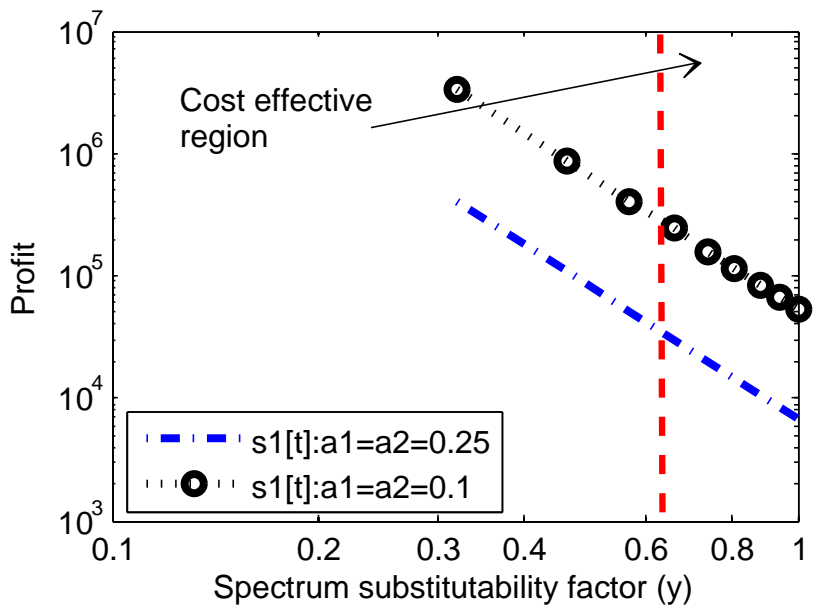

Fig. 3(b)

Fig. 3: (a) Charge at the NE under different spectrum substitutability factor, (b) Profit from secondary service at NE under different spectrum substitutability factor corresponding to the spectrum offered by the primary service.

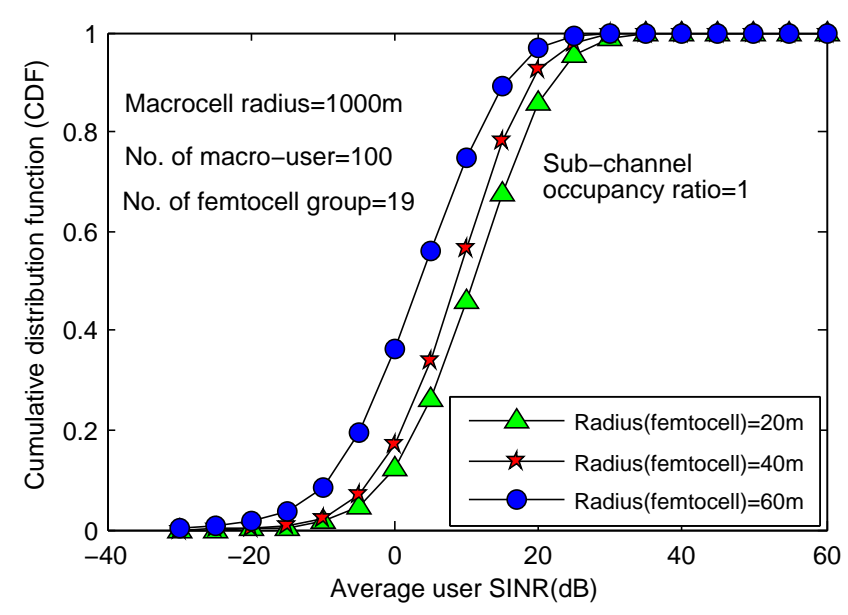

Fig. 4(a)

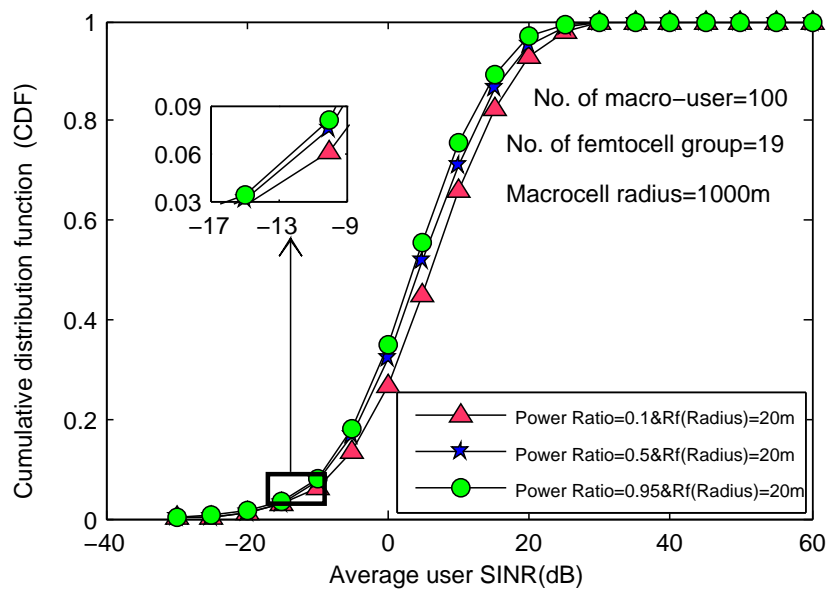

Fig. 4(b)

Fig. 4: (a) CDF of femto users SINR under different femtocell coverage radius, (b) CDF of femto users SINR under different power ratio keeping same coverage radius for femtocell.

will create for the service provider to attract more demand by reducing the spectrum charge.

Fig. 4(a) illustrates the cumulative distribution function (CDF) of user SINR of the three different femtocell radius considerations when the number of macro-users, femtocell cluster and no of users serve by each femtocell are 100, 19 and 10, respectively, and sub-channel occupancy ratio equals to 1 which means the FBS uses all the sub-channels in the operating frequency assignment. The scenario with $20 \mathrm{~m}$ femtocell coverage radius achieves higher femto users SINRs in contrast to the other two scenario where femtocell radius $40 \mathrm{~m}$ and $60 \mathrm{~m}$, respectively. Fig. 4(b) depicts CDF of the SINR of the femto user equipments (FUEs) when the power ratio are $0.1,0.5$ and 0.95 , respectively, and the coverage radius of FBS is $20 \mathrm{~m}$ and the sub-channel usage ratio $\rho_{f}=1$. In contrast to the scenario with the power ratio of 0.1 and 0.5 femtocell resource allocation, the femtocell resource allocation scheme with the power ratio of 0.95 achieves a bit better performance owing to the interference coordination between adjacent FBSs of the same femtocell cluster. The results in Figs. 4(a) and $4(b)$ imply that the femtocell resource allocation scheme used in this paper effectively mitigate the cross layer interference. It can be seen that the proposed model effectively increases the link attainment level of FUs that may be in lower SINR region or upper SINR region. Hence, in contrast to the case of conventional approach this proposed method appreciably protects the near MUs from the cross-tier interference caused by FBSs as shown in Fig. 4(a) and 4(b). This is due to the efficient channel sensing and resource allocation technique identifies explicitly the certified channels, that are unoccupied by neighbour MUs and simultaneously are optimum the FUs, on the entire spectrum. The sub-channel occupancy ratio equals to 1 , which signifies that femtocells fully share the spectrum with the macrocell. This is similarly benefited from avoiding abandoning successive channels by deciding to share one specific channel or not based on the interference level of every channel instead of the total interference level of a cluster of channels. Moreover, it can search for the available resources 


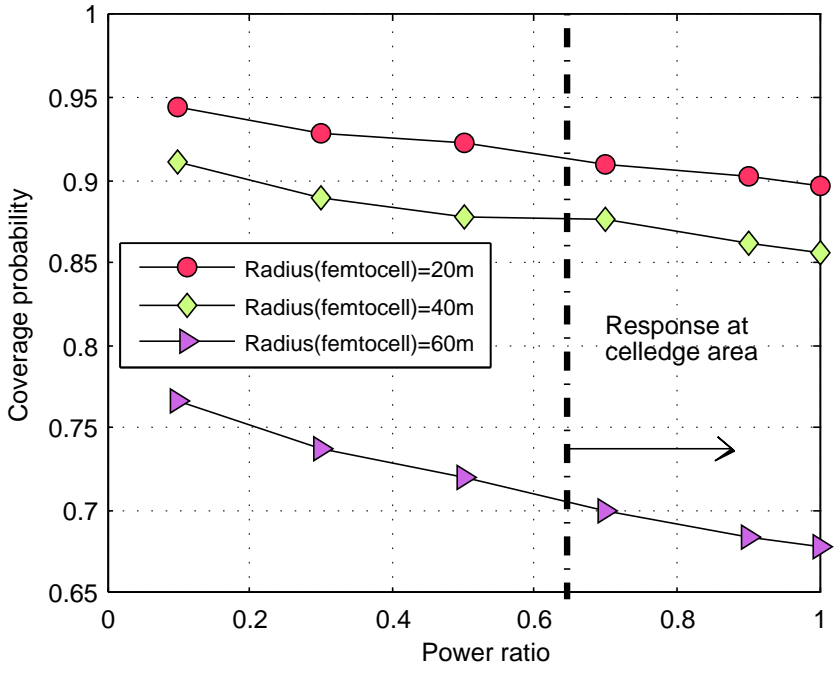

Fig. 5(a)

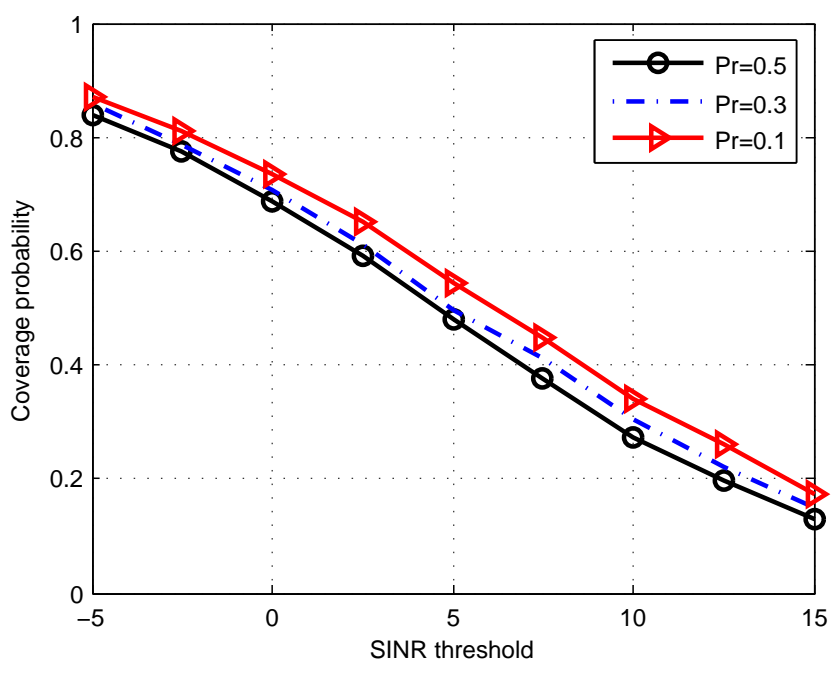

Fig. 5(b)

Fig.5: (a) Coverage probability of mobile users under different femtocell coverage radius, (b) Coverage probability of a mobile users under different SINR threshold.

on the whole system bandwidth.

A portable user is connected to the MBS may be in the coverage of FBS and is unable to connect to that FBS, potentially resulting in cross tier interference. The coverage probability is a possibility that a users SINR is higher than a threshold value likely to happen. In Fig. 5(a), the coverage probability of dualtier networks is shown as a function of power ratio in which different coverage radius of femtocell set for simulation. The coverage probability is decreasing gradually with increase of power ratio for movement of portable users towards the cell edge area. The coverage probability is relatively higher for the scenario where femtocell coverage radius set to $20 \mathrm{~m}$ as compared to other two scenarios where femtocell coverage radius set to $40 \mathrm{~m}$ and $60 \mathrm{~m}$, respectively, at any instant. In Fig. 5(b), the coverage probability of edge users is shown as a function of SINR threshold in which different power ratio factor set for simulation. We see in Fig. 5(b) that the
SINR increases with decrease in power ratio factor for femto tier users. Fig.6 illustrates the overall network throughput for mobile users as a decreasing function of femtocell radius where we articulated on the nature of response at cell edge area. Overall network throughput decreases with increase in power ratio factor where different femtocell radius produces response with different slope. Fig.7 illustrates the overall network throughput as a function of power ratio for proposed scheme, cognitive IM Scheme and traditional scheme respectively. It can be observed that the proposed scheme provides noticeable performance gain over the other schemes. This is mainly due to the fact that the proposed scheme fully reutilizes the spectrum resource applying CR-based resource allocation, whilst effectively protects $\mathrm{MU}$ applying channel sensing.

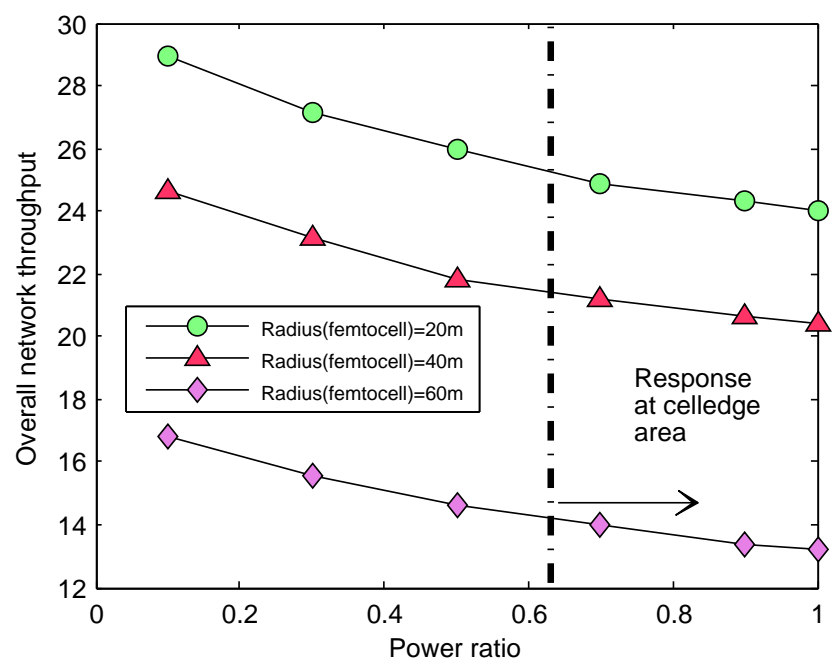

Fig. 6: Dual tier network throughput in mbps under different power ratio factor for a particular femtocell radius

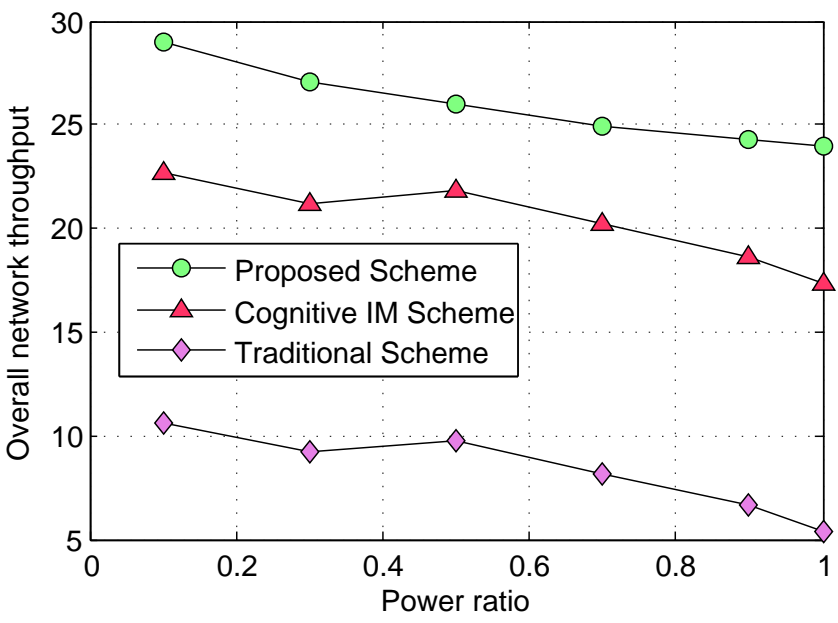

Fig. 7: Dual tier network throughput in mbps under different power ratio factor for different schemes

\section{CONCLUSIONS}

In this paper, we have developed an analytical framework to provide QoS to the users located at macrocell edge area by using non-cooperative game theoretic frequency reuse approach 
in an OFDMA based femtocell networks. The cross-layer interference between the macrocell and femtocells was significantly mitigated with the aid of femtocell resource allocation by spectrum sensing. We have considered the spectrum trading problem in CR networks. In this scenario, number of primary services compete with each other to provide spectrum access facility to the secondary service. This competitive environment has been analyzed by employing a non-cooperative game and the NE has been chosen as the solution. The use of NE is to offer the optimum costing (i.e., optimizes the total profit of all the primary services). For the primary services, the expenditure of sharing the spectrum with the secondary service has been formulated as a function of the QoS deterioration for the primary links. For the secondary service, the channel quality and the spectrum substitutability have been accounted in the demonstrated model.

\section{REFERENCES}

[1] M D Weng, B H Lee, and J M Chen, "Two novel price-based algorithms for spectrum sharing in cognitive radio networks," Eurasip Journal Wireless Communications and Networking.

[2] S. Maharjan, Y. Zhang, and S. Gjessing, "Economic Approaches for Cognitive Radio Networks: A Survey," Wireless Pers. Commun., vol. 57, no. 1, pp. 3351, Mar. 2011

[3] L. S. Shapley,"Stochastic Games," PNAS, vol. 39, no. 10, pp. 10951100,Oct. 1953.

[4] Z. Han, Z. Ji, and K. J. R. Liu, "Non-cooperative resource competition game by virtual referee in multi-cell OFDMA networks," IEEE J. on Sel. Areas in Commun., vol. 25,no. 6, pp. 1079 1090, Aug. 2007.

[5] A. T. Hoang, and Y.-C. Liang, "Maximizing spectrum utilization of cognitive radio networks using channel allocation and power control," In IEEE VTC, 2006.

[6] Thomas David Novlan, Radha Krishna Ganti, Arunava Ghosh, Jeffrey G. Andrews, "Analytical Evaluation of Fractional Frequency Reuse for Heterogeneous Cellular Networks," IEEE Transactions on Communications, Vol.60,No.7, July 2012

[7] A. T. Hoang, Y. C. Liang and M. H. Islam, "Maximizing Throughput of Cognitive Radio Networks with Limited Primary Users Cooperation," in Proc. IEEE ICC, June 2007, pp. 51775182.

[8] Lay Teen Ong, Mohammad Shikh-Bahaei, and Jonathon A. Chambers "Variable rate and variable power MQAM system based on Bayesian bit error rate and channel estimation techniques," IEEE Transaction on Communications, Vol.56,No.2,Feb2008.

[9] H. N. Agiza, G.-I. Bischi, and M. Kopel, "Multistability in a dynamic cournot game with three oligopolists," Mathematics and Computers in Simulation, vol. 51, pp. 63-90, 1999

[10] A. Hald, Statistical Tables and Formulas. New York: Wiley, 1952

[11] G.I. Bischi and A. Naimzada,"Global analysis of a dynamic duopoly game with bounded rationality," In Advances in Dynamic Games and Applications, Vol. 5., Birkhaur, Boston (1999).

[12] Dong-Chan Oh and Yong-Hwan Lee, "Cognitive Radio Based Resource Allocation in Femto-cell," Journal of Communications and Networks, Vol.14, No.3, June 2012.

[13] Vu Nguyen Ha and Long Bao Le, "Distributed Base Station Association and Power Control for Heterogeneous Cellular Networks," IEEE Transactions on Vehicular Technology, Vol.63, Issue 1, July 2013.

[14] Duy Trong Ngo, Long Bao Le and Tho Le-Ngoc,"Distributed ParetoOptimal Power Control for Utility Maximization in Femtocell Networks," IEEE Transactions on Wireless Communications, Vol.11, No 10, Oct.2012.
[15] Dusit Niyato, Ekram Hossain,"Market-Equilibrium, Competitive, and Cooperative Pricing for Spectrum Sharing in Cognitive Radio Networks: Analysis and Comparison," IEEE Transactions on Communications, Vol.7, No.11, Nov 2008.

[16] Dusit Niyato, Ekram Hossain,"Competitive Pricing for Spectum Sharing in Cognitive Radio Networks: Dynamic Game, Inefficiency of Nash Equilibrium, and Collusion" IEEE Journal on Selected Areas in Communications, Vol.26, No.1, Jan 2008.

[17] Xinbing Wang, Zheng Li, Pengchao Xu, Youyun Xu, Xinbo Gao, and Hsiao-Hwa Chen, "Spectrum Sharing in Cognitive Radio Networks- An Auction Based Approach," IEEE Transaction on Systems, Man, and Sybernetics-Part B.

[18] Zhikun Xu, Geoffrey Ye Li, Chenyang Yang and Xiaolong Zhu, "Throughput and Optimal Threshold for FFR Schemes in OFDMA Cellular Networks," IEEE Transactions On Wireless Communications, Vol. 11, No. 8, August 2012.

[19] J Ghosh, S Dhar Roy,"Qualitative analysis for coverage probability and energy efficiency in cognitive- femtocell networks under macrocell infrastructure," ELECTRONICS LETTERS, 20th August 2015, Vol. 51, No. 17, pp. 13781380.

[20] Jane Wei Huang and Vikram Krishnamurthy,"Cognitive Base Stations in LTE/3GPP Femtocells: A Correlated Equilibrium Game-Theoretic Approach," IEEE Transactions on Communications,Vol.59,No.12,December 2011.

[21] H Lee, K Han, Y Hwang and S Choi,"Opportunistic band sharing for point-to-point link connection of cognitive radios," in 4th International Conference on Cognitive Radio Oriented Wireless Networks and Communiactions (CROWNCOM09), pp. 16, June 2009.

[22] Joydev Ghosh, Varsha Panjwani, Sanjay Dhar Roy,"FemtocellThroughput analysis in OFDMA-CR Networks," IJEIC, Vol.6, Issue.6 (2015),pp.9-18.

[23] Ying-Chang Liang, Yonghong Zeng, Edward C.Y. Peh, Anh Tuan Hoang, "Sensing-Throughput Tradeoff for Cognitive radio Networks," IEEE Transactions on Wireless Communications, Vol.7, No.4, April 2008.

[24] J. Ghosh, S. Dhar Roy,"Mitigating ICI at Cell Edges in CognitiveFemtocell Networks Through Fractional Frequency Reuse," IJCNDS, Vol.16, No.2, Jan.2016.

[25] A. Al-Dulaimi, et. al. "Adaptive Management of Cognitive Radio Networks Employing Femtocells", IEEE Systems Journal, Vol. 11, Issue 4, pp. 2687-2698, Dec 2017

[26] Q. Ni, C. Zarakovitis. "Nash Bargaining Game Theoretic Scheduling for Joint Channel and Power Allocation in Cognitive Radio Systems", IEEE Journal on Selected Areas in Communications, Vol. 30, No. 1, Jan 2012, pp. 70-81.

[27] Z. Song, et. al. "On the Spectral-Energy Efficiency and Rate Fairness Tradeoff in Relay-Aided Cooperative OFDMA Systems", IEEE Transactions on Wireless Communications, Vol. 15, Issue 9, pp. 6342 6355, Sept. 2016.

[28] Y. Hao, et. al. On the Energy and Spectral Efficiency Tradeoff in Massive MIMO-Enabled HetNets with Capacity-Constrained Backhaul Links, IEEE Transactions on Communications, Vol. 65, Issue 11, pp. 4720 4733, November 2017.

[29] C. Yang, et al. "Interference-aware energy efficiency maximization in 5G ultra-dense networks", IEEE Transactions on Communications, vol. 65, issue 2, pp. 728-739, Feb. 2017.

[30] Junfei Qiu, Qihui Wu, Yuhua Xu, Youming Sun and Ducheng Wu,"Demand-aware resource allocation for ultra-dense small cell networks: an interference-separation clustering-based solution,'TRANSACTIONS ON EMERGING TELECOMMUNICATIONS TECHNOLOGIES, Volume 27, Issue 8, August 2016, Pages: 1071 -1086 .

[31] lvaro Ricieri Castro e Souza, Jos Roberto de Almeida Amazonas and Taufik Abro,"Energy-efficiency maximisation for cooperative and noncooperative OFDMA cellular networksa survey,"TRANSACTIONS ON EMERGING TELECOMMUNICATIONS TECHNOLOGIES, Volume 27, Issue 2, February 2016, Pages: 216 - 248 